\title{
Sandpile model on the Sierpinski gasket fractal
}

\author{
Brigita Kutnjak-Urbanc, ${ }^{1}$ Stefano Zapperi, ${ }^{1}$ Sava Milošević, ${ }^{2}$ and H. Eugene Stanley ${ }^{1}$ \\ ${ }^{1}$ Center for Polymer Studies and Department of Physics, Boston University, Boston, Massachusetts 02215 \\ ${ }^{2}$ Faculty of Physics, University of Belgrade, P.O. Box 368, 11001 Belgrade, Yugoslavia
}

(Received 14 February 1996)

\begin{abstract}
We investigate the sandpile model on the two-dimensional Sierpinski gasket fractal. We find that the model displays interesting critical behavior, and we analyze the distribution functions of avalanche sizes, lifetimes, and topplings and calculate the associated critical exponents $\tau=1.51 \pm 0.04, \alpha=1.63 \pm 0.04$, and $\mu=1.36 \pm 0.04$. The avalanche size distribution shows power-law behavior modulated by logarithmic oscillations which can be related to the discrete scale invariance of the underlying lattice. Such a distribution can be formally described by introducing a complex scaling exponent $\tau^{*} \equiv \tau+i \delta$, where the real part $\tau$ corresponds to the power law and the imaginary part $\delta$ is related to the period of the logarithmic oscillations. [S1063-651X(96)03907-4]
\end{abstract}

PACS number(s): 64.60.Ak, 02.60.Cb

\section{INTRODUCTION}

The concept of self-organized criticality (SOC) has been introduced by Bak et al. [1] to describe the tendency of a large class of dynamical systems to spontaneously evolve into a critical state without fine tuning of any external parameter. Sandpile models $[1,2]$ have been introduced as an example of this kind of phenomena and have been widely studied numerically and analytically [3-7]. Two principal analytical approaches have been followed: the first involves the group theory formalism introduced by Dhar and coworkers [8] and the second is a real-space renormalization scheme recently developed by Pietronero et al. [9]. Other theoretical approaches involve nonlinear continuous differential equations [10,11].

Sandpile models are inspired by the dynamics of sand flowing along the slope of a pile. By adding sand grains to the pile the system eventually reaches a stationary state characterized by avalanches of all length scales. The term criticality refers here to the absence of any characteristic length scale in this state. Sandpile models have been studied mostly on Euclidean lattices. It has been shown that different kinds of Euclidean lattices do not affect the critical exponents [4]. This fact is similar to the universality observed in ordinary critical phenomena. Moreover, in the case of the Bethe lattice one recovers the mean field results $[12,13]$. However, sandpile models, to our knowledge, have not been studied on a fractal substrate, in particular on a simple deterministic fractal such as that epitomized by the Sierpinski gasket (SG).

It has been shown, via specific calculations [14] and through general rigorous analysis [15], that for the standard Ising model (and for some more general models) on finitely ramified fractals no spontaneous magnetization can exist at any finite temperature. It might have happened that, by some assumed analogy, no self-organized critical behavior has been expected so far to occur on the deterministic fractals. However, we shall demonstrate that the SOC phenomenon exists on the SG fractal and displays interesting features. Specifically, we study numerically the critical height sandpile model on the SG lattice with the generator scaling base $b=2$ which corresponds to the fractal dimension

$D=\ln 3 / \ln 2 \approx 1.58$. We calculate the distribution of avalanche sizes, their lifetimes, and topplings. The avalanche size distribution shows a power-law behavior modulated by logarithmic oscillations. This kind of oscillation has already been observed in the scaling functions of different systems [16], and here it can be related to the discrete scale invariant nature of the underlying fractal lattice. It is interesting to note that complex scaling exponents have been recently detected in earthquake statistics [17].

The measured scaling exponents vary with the system size $L$ and the values, extrapolated to $L \rightarrow \infty$, appear to differ from those computed on the Euclidean lattices. Computing expectation values, we are able to verify the relationships between different critical exponents.

In addition, we investigate time correlations of the number of drops and topplings during the avalanche. Calculating the power spectra, we find that as in the case of the twodimensional Euclidean lattice $[18,19]$ there are no long-range temporal correlations.

\section{THE MODEL}

Our cellular automata model is defined on the SG lattice as shown in Fig. 1. The number $n$ is related to the number of sites $L=2^{n}+1$ along one direction of the lattice and is used hereafter as a measure of the system size. Within the sandpile model, all the sites of the fractal lattice are exposed to the same local dynamical rules. The exceptions are the three apex sites where the sand grains flow out of the system. The dynamics begins when we associate an integer height variable $z_{i}$ with every lattice site $i$. At each later step one lattice site is chosen at random and its height is increased by one. Whenever the height on a site $i$ reaches the critical value $z_{c}=4$, the site becomes unstable (active) and relaxes according to the following rules

$$
\begin{aligned}
& z_{i} \rightarrow z_{i}-4, \\
& z_{j} \rightarrow z_{j}+1,
\end{aligned}
$$



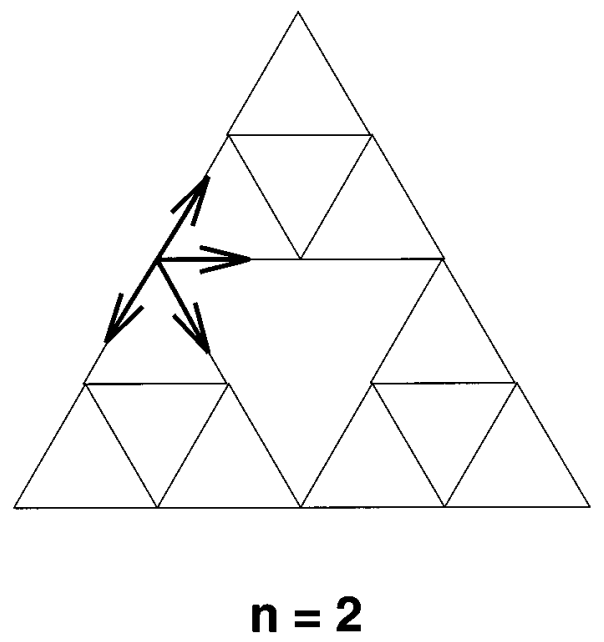

FIG. 1. An example of the SG lattice with the generator $b=2$, at the stage of construction $n=2$, and with linear size $L=5$. Each site has four neighbors except for the three apex sites with only two neighboring sites. The arrows indicate the direction of sand flow from a chosen site.

where $j$ are the nearest neighbors of $i$. These rules conserve the total number of grains, except on the three apex sites (independent of the system size) where two sand grains are lost. Successive relaxation events generate the sand flow that eventually brings the sand out of the system. Due to the local conservation, imposed by the dynamical rules, the system finally evolves into the stationary state characterized by the balance between the input and the output flow.

The critical exponents are extracted from avalanche distributions in the stationary state. We define the size $S$ as the number of distinct sites visited by an avalanche, the toppling size $m$ as the number of relaxation events, and the lifetime $T$ as the number of updating steps during an avalanche. All these quantities are expected to be distributed as power laws

$$
\begin{gathered}
P(S) \sim S^{-\tau}, \\
P(m) \sim m^{-\mu}, \\
P(T) \sim T^{-\alpha},
\end{gathered}
$$

where $\tau, \mu$, and $\alpha$ are critical exponents of the respective distribution functions $P(S), P(m)$, and $P(T)$. We can relate these exponents by considering conditional expectation values for an average avalanche size $\left\langle S_{T}\right\rangle$ and an average toppling size $\left\langle m_{T}\right\rangle$ at a given avalanche lifetime $T$ :

$$
\begin{aligned}
& \left\langle S_{T}\right\rangle \sim T^{\beta}, \\
& \left\langle m_{T}\right\rangle \sim T^{\gamma},
\end{aligned}
$$

and similar other relations. By taking into account the definitions of critical exponents, given by Eqs. (3)-(7), scaling relations between exponents can be derived [6]:

$$
\begin{aligned}
& \tau=1+(\alpha-1) / \beta, \\
& \mu=1+(\alpha-1) / \gamma .
\end{aligned}
$$

TABLE I. The fraction of sites having height equal to $z=1,2$, 3 , and 4 and the average height $\langle z\rangle$ as found for different system sizes $L=2^{n}+1$ and $n=3,4,5,6$, and 7 .

\begin{tabular}{cccccc}
\hline \hline$n$ & $p_{1}$ & $p_{2}$ & $p_{3}$ & $p_{4}$ & $\langle z\rangle$ \\
\hline 3 & 0.075 & 0.143 & 0.306 & 0.476 & 3.18 \\
4 & 0.070 & 0.136 & 0.302 & 0.492 & 3.22 \\
5 & 0.069 & 0.133 & 0.299 & 0.499 & 3.23 \\
6 & 0.068 & 0.132 & 0.298 & 0.501 & 3.23 \\
7 & 0.068 & 0.132 & 0.298 & 0.502 & 3.23 \\
\hline \hline
\end{tabular}

Finally we study temporal correlations by considering the number of active sites and the number of grains falling out of the system at each time step. The power spectrum of this signal falls off as

$$
S(f) \sim f^{-\phi} .
$$

In the Euclidean case, $\phi=2$ showing the absence of longrange temporal correlations.

\section{SIMULATION RESULTS}

We perform numerical simulations for different lattice sizes ranging from $n=3$ to $n=7$. The total number of sites $S_{n+1}$ of the system size $n+1$ is related to the number of sites $S_{n}$ of the system size $n$ via the equation $S_{n+1}=3 S_{n}-3$ with $S_{0}=3$, which corresponds to a total number of lattice sites going from $S_{3}=42$ to $S_{7}=3282$.

A simple way to characterize the properties of the stationary state is to compute the fraction $p_{z}$ of sites having height $z_{i}=1,2,3$, and 4 . We report these results in Table I for different system sizes, together with an average height $\langle z\rangle$. The obtained values are very close to those found on the Euclidean lattice $[4,5]$.

In Fig. 2 we show the avalanche size distribution for different system sizes. One can see that there are quite a few avalanches (represented by the last peak of each distribution

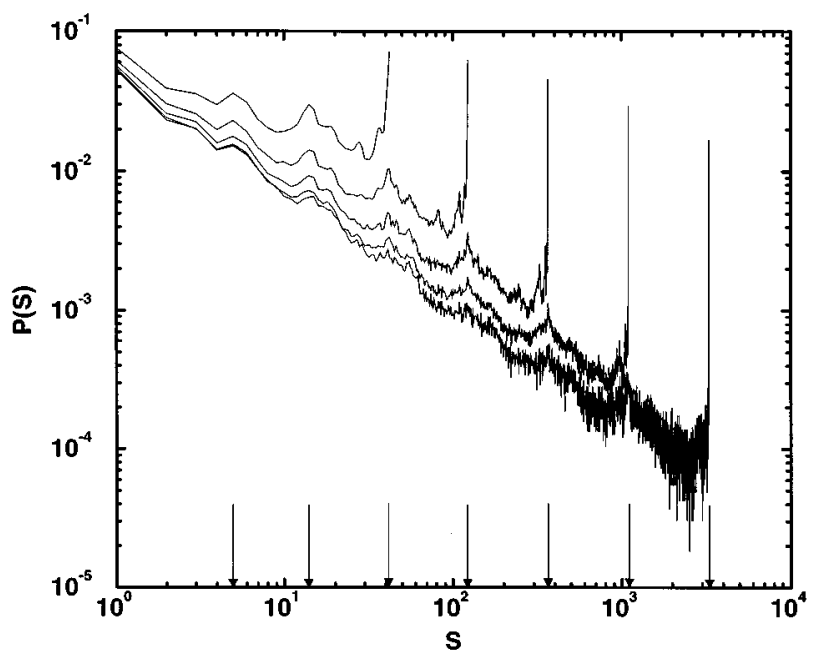

FIG. 2. The distribution of avalanche sizes of the sandpile model on a SG lattice. Different curves correspond to different system sizes. The arrows indicate the peaks in the distribution. 
(a)

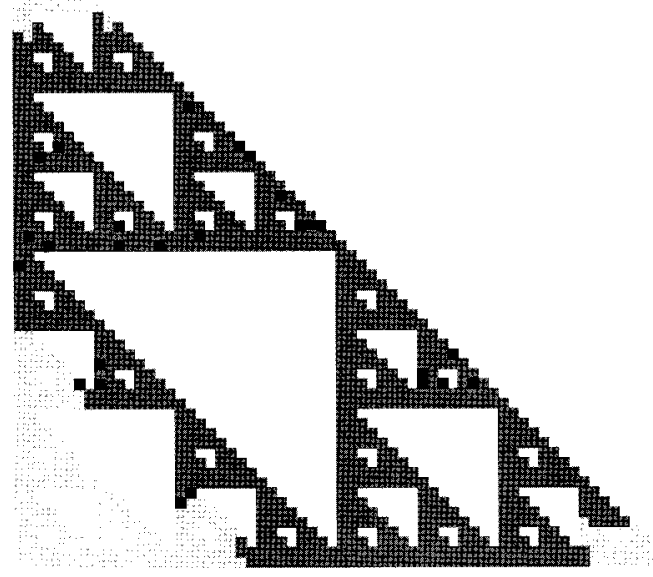

(b)

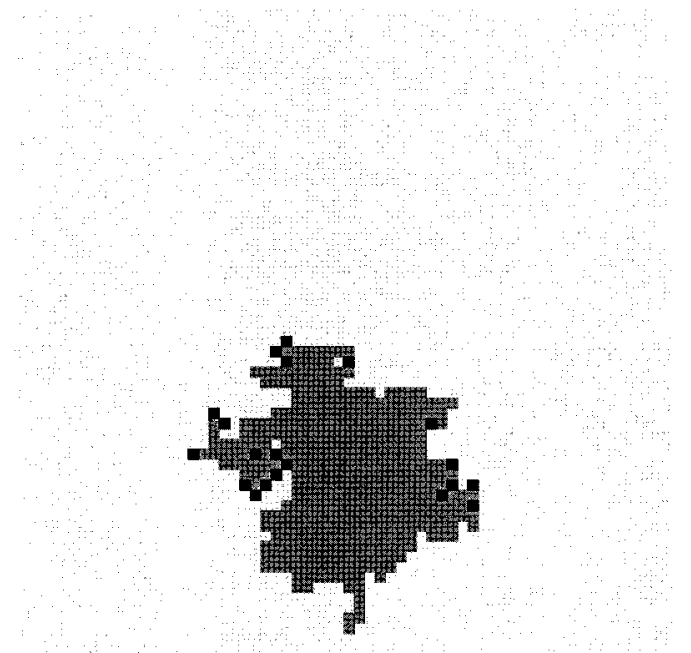

FIG. 3. A snapshot of an avalanche on the Sierpinski gasket lattice (a) compared with an avalanche on the Euclidean square lattice (b). Active sites are depicted in black and sites that have toppled at least once are colored in gray.

curve) which span the entire lattice. This occurs because the balance between incoming and outgoing particles forces the avalanches to reach the three apex sites. Due to the selfsimilarity of the underlying lattice, the same phenomenon occurs on all fractal substructures, which is manifested by a series of peaks on each distribution curve.

The phenomenon described above is reflected in a peculiar behavior of the active sites during the evolution of an avalanche: the active sites are localized (trapped) within fractal substructures for many time steps. Such a trapping does not occur in the Euclidean lattice where the active sites are essentially on the avalanche front. This is apparent from Fig. 3 where the active sites for a typical avalanche in the Euclidean square lattice are compared with an avalanche on the fractal lattice. Similar differences which spring from differences in the topology of the lattices were noted before [20] in a study of linear polymers on the diamond hierarchical lattice.

The power-law behavior in a double logarithmic plot is

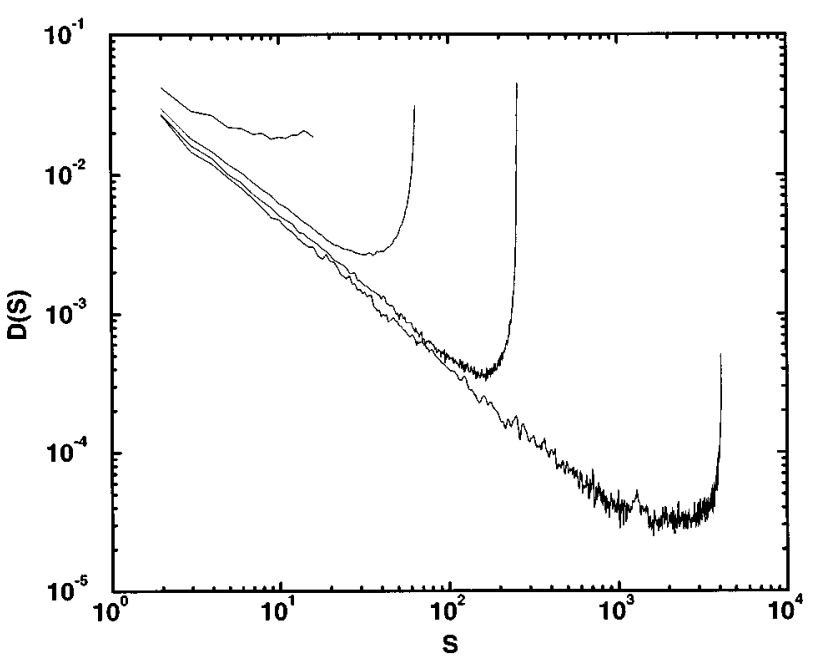

FIG. 4. The distributions of avalanche sizes for a sandpile model on a square lattice with four exiting points are depicted for several system sizes $L=4,8,16$, and 32 . Data for $L=16$ and $L=32$ are averaged at sizes $S>50$.

modulated by oscillations with a period $p$ that can be related to the scaling properties of the SG lattice. A self-similar lattice is left invariant only by a discrete set of scale transformations, namely, by those with a scaling parameter of the form $\lambda=b^{n}$. Under this condition, it has been shown [21] that the most general scale invariant function of the realspace coordinates is a power-law multiplied by a logarithmically periodic function. These oscillations can be formally described by introducing a complex scaling exponent $\tau^{*} \equiv \tau+i \delta$ where the real part $\tau$ corresponds to the powerlaw exponent, while the imaginary part $\delta$ is related to the period of oscillations. In our case $\delta=2 \pi / p=2 \pi /$ $\ln 3=5.72$. To extract $\tau$, we fit the distribution with a power law modulated by a periodic function.

The last peak in the distribution of avalanche sizes is a consequence of the fact that at any system size there are only three boundary points where the sand can flow out of the system, in contrast to the Euclidean lattice where the number of boundary points increase in proportion to the system size. However, one can study the effect of boundaries by calculating the avalanche size distribution of the same sandpile model on the Euclidean lattice with only four boundary points, e.g., the four corner sites of the square lattice (for the rest of the edge points periodic boundary conditions apply). The results are shown in Fig. 4 for system sizes $L=4,8$, 16, and 32. The distributions are power laws with peaks at the total numbers of sites on the lattice.

In the SG case, we report in Figs. 5 and 6 the distributions of avalanche lifetimes and topplings in a double logarithmic plot. Both distributions display pure power-law behavior without any modulations. The power-law regimes grow with the system size.

As in the case of the Euclidean lattice [4], the scaling exponents depend on the system size. We can, however, extract the asymptotic results by plotting the logarithm of the exponents versus $1 / \log _{10} L$, where $L$ is the linear size of the lattice. This relationship is presented in Fig. 7, where we 


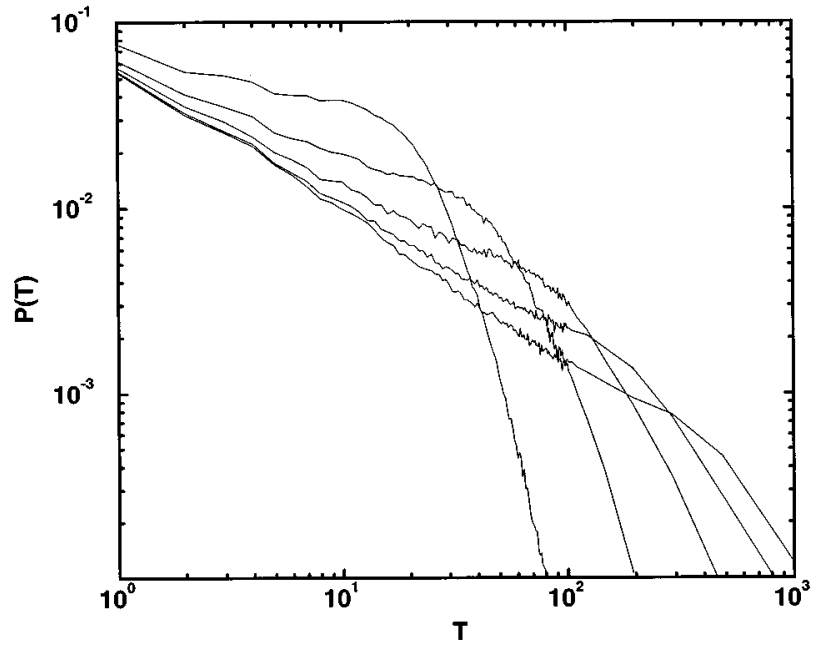

FIG. 5. The distribution of avalanche lifetimes of the sandpile model on a SG lattice as calculated for different system sizes. Data are logarithmically binned at lifetimes $T>50$.

depict also the extrapolated critical exponents in the limit $n \rightarrow \infty$. We found the extrapolated values $\tau_{\infty}=1.51 \pm 0.04$, $\alpha_{\infty}=1.64 \pm 0.04$, and $\mu_{\infty}=1.36 \pm 0.04$ for the avalanche size, lifetime and toppling distributions, respectively. The distribution functions presented in these figures have been calculated by averaging over $2^{17}$ avalanches.

To check the consistency of our results we compute the scaling exponents of the conditional expectation values, i.e., exponents related to the average avalanche size $\left\langle S_{T}\right\rangle$ and number of topplings $\left\langle m_{T}\right\rangle$ in dependence on the lifetime $T$. Figure 8 shows results of our computation in a double logarithmic plot. The slopes in the figure correspond to the exponents $\beta$ and $\gamma$ as defined in Eqs. (6) and (7) and are found to be $\beta=1.13 \pm 0.05$ and $\gamma=1.73 \pm 0.05$. These two values can be compared with the ones evaluated from the scaling

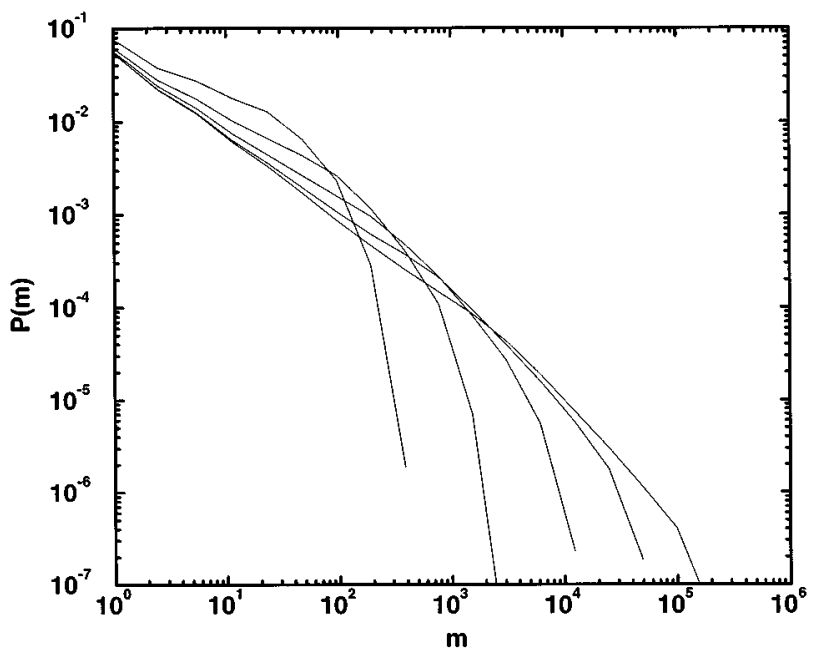

FIG. 6. The distribution of the number of topplings per avalanche of the sandpile model on a SG lattice as calculated for different system sizes. Data are logarithmically binned at toppling sizes $m>50$.

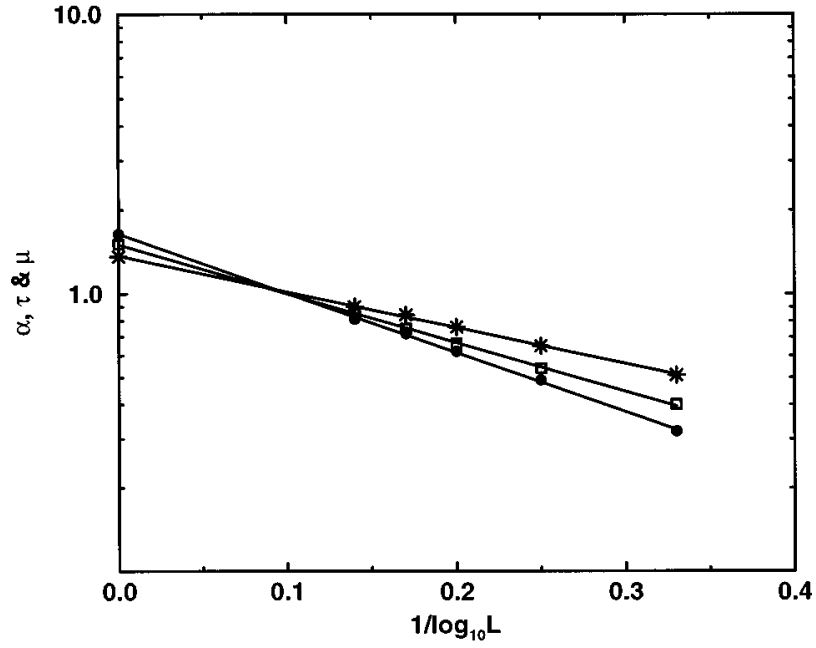

FIG. 7. The logarithm of the critical exponents found as a result of a simulation for different lattice sizes plotted against $1 / \log _{10} L$. Estimations of the three exponents in the limit of infinitely large lattice size $\left(1 / \log _{10} L \rightarrow 0\right)$ are also shown. The extrapolated exponents in the limit $L \rightarrow \infty$ are $\tau_{\infty}=1.51 \pm 0.04, \alpha_{\infty}=1.63 \pm 0.04$, and $\mu_{\infty}=1.36 \pm 0.04$

relations given by Eqs. (8) and (9), and using the estimated critical exponents $\tau_{\infty}, \alpha_{\infty}$, and $\mu_{\infty}$. Thus we find the values $\beta=1.24 \pm 0.12$ and $\gamma=1.75 \pm 0.18$, which are in agreement, within the numerical error, with the directly obtained values from Fig. 8.

Finally, within the scope of the sandpile model [1], we calculate the temporal correlations of two quantities: the number of particles which fall out of a system in a unit time and the number of topplings. The unit time in this case corresponds to one updating step of the lattice variables. We calculate the power spectra of the above two quantities. In

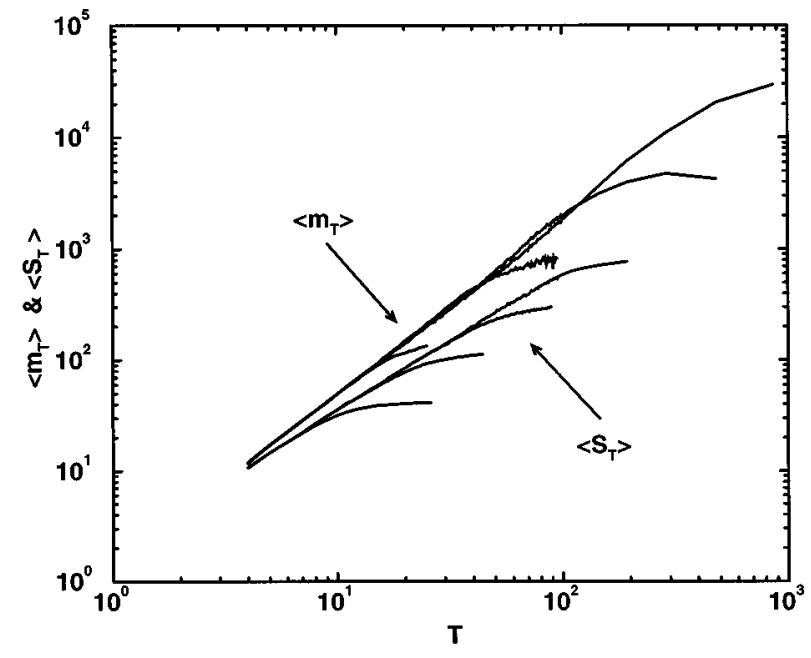

FIG. 8. The average avalanche size $\left\langle S_{T}\right\rangle$ and the average number of topplings $\left\langle m_{T}\right\rangle$ as functions of the lifetime $T$, presented in a double logarithmic plot. The corresponding slopes are $\beta=1.13 \pm 0.05$ and $\gamma=1.75 \pm 0.05$. Data are logarithmically binned at lifetimes $T>50$. 


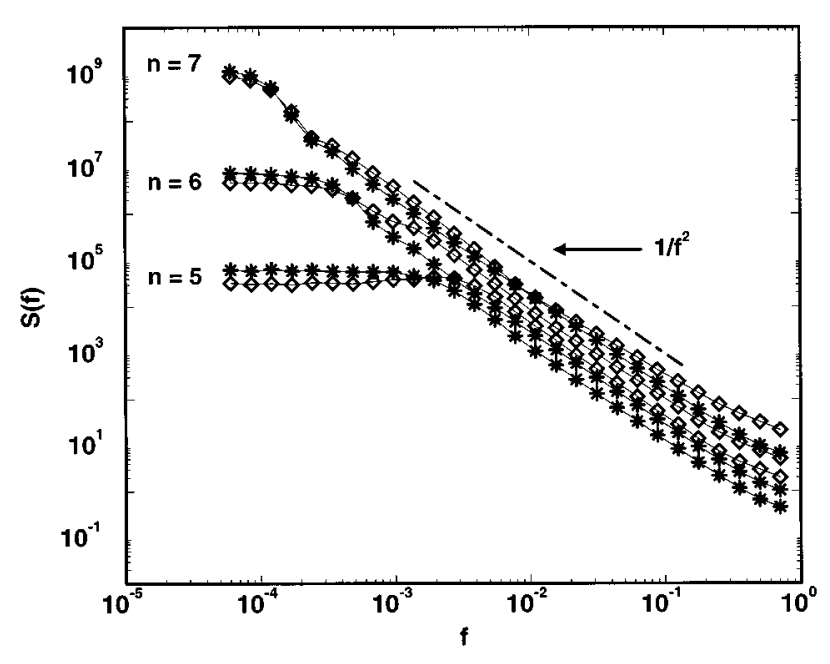

FIG. 9. Power spectra of the number of particles which drop out of the system (stars) and of the number of topplings (diamonds) in a given time step. The data for three system sizes are depicted, from $n=5$ to $n=7$. The results show $1 / f^{2}$ type of spectra, which indicates an exponential decay of time correlations. It can be observed that the frequency at which the $1 / f^{2}$ type of the spectrum crosses over to the white noise type (i.e., the flat part of the spectrum), due to the finite size of the system, decreases with the lattice size.

both cases we find a $1 / f^{2}$ type of spectrum. Thereby, the type of temporal correlations turns out to be the same as in the original model on a two-dimensional square lattice $[18,19]$. Our results are presented in Fig. 9. The flattening of the $1 / f^{2}$ spectrum at small frequencies is due to finite size effects. In contrast to other scaling exponents presented in this paper, the exponents of power spectra do not vary with the system size, that is, the $1 / f^{2}$ type of spectrum corresponds to an exponential decay of temporal correlations independently of the system size.

\section{CONCLUSION}

We computed numerically the scaling exponents for the avalanche distributions in the critical height sandpile model on the Sierpinski gasket lattice with the generator base $b=2$. The lattice coordination number is the same as in the two-dimensional square lattice and therefore the dynamical rules of the sandpile model are exactly the same. The boundaries, however, are different, since on the SG lattice the sand can flow out only through three sites at every scale. This fact substantially changes the avalanche dynamics. The active sites become trapped (localized) and topple more than once during a single avalanche.

In relation to the standard critical phenomena it is interesting to note how the dimensionality and the topology of the lattice affect the critical behavior of the model. In one dimension, the critical height sandpile model is trivial in that avalanches are not power-law distributed [1]. A similar behavior occurs, for example, in the Ising model where no phase transition is observed in dimension less than two. We have shown, however, that on a finitely ramified fractal, the sandpile has nontrivial critical behavior, in contrast to the Ising model which has no phase transition on such a fractal lattice $[14,15]$.

Finally, we note that self-similar lattices have been proven very helpful in constructing exact real-space renormalization group transformations [22,23] for standard critical phenomena. Having demonstrated that self-organized criticality can exist on a fractal lattice, it would be beneficial to find such a transformation for this model, trying to link the rigorous approach of Dhar and co-workers [8] with the realspace renormalization scheme presented in $[9,24]$.

Note added in proof: After finishing this work we came to know about recent observations of complex critical exponents; we thank D. Sornette [25] for calling these to our attention.

\section{ACKNOWLEDGMENTS}

This work was supported by the National Science Foundation. B.K.-U. acknowledges additional financial support from the Ministry of Science of Slovenia. We would like to thank S. Havlin, K. B. Lauritsen, H. Leschhorn, and L. Pietronero for useful discussions and comments.
[1] P. Bak, C. Tang, and K. Wiesenfeld, Phys. Rev. Lett. 59, 381 (1987); Phys. Rev. A 38, 364 (1988).

[2] Y. C. Zhang, Phys. Rev. Lett. 63, 470 (1989).

[3] L. P. Kadanoff, S. R. Nagel, L. Wu, and S. Zhu, Phys. Rev. A 39, 6524 (1989).

[4] S. S. Manna, J. Stat. Phys. 59, 509 (1990); Physica A 179, 249 (1991).

[5] V. B. Priezzhev, J. Stat. Phys. 74, 955 (1994).

[6] K. Christensen, H. C. Fogedby, and H. J. Jensen, J. Stat. Phys. 61, 653 (1991).

[7] P. Grassberger and S. S. Manna, J. Phys. (Paris) 51, 1077 (1990).

[8] D. Dhar and R. Ramaswamy, Phys. Rev. Lett. 63, 1659 (1989); D. Dhar, ibid. 64, 1613 (1991); S. N. Majumdar and D.
Dhar, Physica A 185, 129 (1992).

[9] L. Pietronero, A. Vespignani, and S. Zapperi, Phys. Rev. Lett.

72, 1690 (1994); Phys. Rev. E 51, 1711 (1995).

[10] A. Diaz-Guilera, Phys. Rev. A 45, 8551 (1992).

[11] T. Hwa and M. Kardar, Phys. Rev. Lett. 62, 1813 (1989).

[12] D. Dhar and S. N. Majumdar, J. Phys. A 23, 4333 (1990).

[13] C. Tang and P. Bak, J. Stat. Phys. 51, 797 (1988).

[14] Y. Gefen, A. Aharony, Y. Shapir, and B. B. Mandelbrot, J. Phys. A 17, 435 (1984); S. H. Liu, Phys. Rev. B 32, 5804 (1985); J. H. Luscombe and R. C. Desai, ibid. 32, 1614 (1985); S. Bhattacharya, Physics Lett. 112A, 51 (1985); T. Stošić, B. Stošić, S. Milošević, and H. E. Stanley, Phys. Rev. E 49, 1009 (1994).

[15] D. Cassi, Phys. Rev. Lett. 68, 3631 (1992); Physica A 191, 549 
(1992); D. Cassi and S. Regina, Phys. Rev. Lett. 70, 1647 (1993); Y. Shi and C. D. Gong, Phys. Rev. E 49, 99 (1994).

[16] B. Doucot et al., Phys. Rev. Lett. 57, 1235 (1986).

[17] D. Sornette and C. G. Sammis, J. Phys. (France) I 5, 607 (1995); W. I. Newman, D. L. Turcotte, and A. M. Gabrielov, Phys. Rev. E 52, 4827 (1995).

[18] J. Kertész and L. B. Kiss, J. Phys. A 23, L433 (1990).

[19] H. J. Jensen, K. Christensen, and H. C. Fogedby, Phys. Rev. B 40, R7425 (1989).

[20] M. Kaufman, T. Berger, P. D. Gujrati, and D. Bowman, Phys. Rev. A 41, 4371 (1990).

[21] D. Bessis, J. S. Geronimo, and P. Moussa, J. Phys. (Paris) Lett.
44, 977 (1983); L. Pietronero, in Order and Chaos in Nonlinear Physical Systems, edited by S. Lundqvist, N. H. March, and M. Tosi (Plenum Publishing Corporation, New York, 1988).

[22] D. Dhar, J. Math. Phys. 18, 577 (1977); 19, 5 (1978).

[23] M. Kaufman and R. B. Griffiths, Phys. Rev. B 30, 244 (1984).

[24] Some progress in linking the two approaches has been recently presented in E. V. Ivashkevich, Phys. Rev. Lett. 75, 465 (1995).

[25] J.-C. Anifrani, C. LeFloch, D. Sornette, and B. Souillard, J. Phys. I (France) 5, 631 (1995); H. Saleur and D. Sornette, ibid. 6, 327 (1996); D. Sornette, A. Johansen, and J.-P. Bouchaud, ibid. 6, 167 (1996). 